\title{
Structural and functional characterization of MERS coronavirus papain-like protease
}

\author{
Min-Han Lin ${ }^{1}$, Shang-Ju Chuang ${ }^{1}$, Chiao-Che Chen ${ }^{1}$, Shu-Chun Cheng ${ }^{1}$, Kai-Wen Cheng ${ }^{1}$, Chao-Hsiung Lin ${ }^{1}$, \\ Chiao-Yin Sun ${ }^{2^{*}}$ and Chi-Yuan Chou ${ }^{1 *}$
}

\begin{abstract}
Backgrounds: A new highly pathogenic human coronavirus (CoV), Middle East respiratory syndrome coronavirus (MERS-CoV), has emerged in Jeddah and Saudi Arabia and quickly spread to some European countries since September 2012. Until 15 May 2014, it has infected at least 572 people with a fatality rate of about 30\% globally. Studies to understand the virus and to develop antiviral drugs or therapy are necessary and urgent. In the present study, MERS-CoV papain-like protease (PL ${ }^{\text {pro }}$ ) is expressed, and its structural and functional consequences are elucidated.

Results: Circular dichroism and Tyr/Trp fluorescence analyses indicated that the secondary and tertiary structure of MERS-CoV PL ${ }^{\text {pro }}$ is well organized and folded. Analytical ultracentrifugation analyses demonstrated that MERS-CoV PL ${ }^{\text {pro }}$ is a monomer in solution. The steady-state kinetic and deubiquitination activity assays indicated that MERS-CoV PL ${ }^{\text {pro }}$ exhibits potent deubiquitination activity but lower proteolytic activity, compared with SARS-CoV PL ${ }^{\text {pro }}$. A natural mutation, Leu105, is the major reason for this difference.

Conclusions: Overall, MERS-CoV PL pro bound by an endogenous metal ion shows a folded structure and potent proteolytic and deubiquitination activity. These findings provide important insights into the structural and functional properties of coronaviral PL ${ }^{\text {pro }}$ family, which is applicable to develop strategies inhibiting PL ${ }^{\text {pro }}$ against highly pathogenic coronaviruses.
\end{abstract}

Keywords: MERS coronavirus, Papain-like protease, Deubiquitination, Antiviral target

\section{Background}

In September 2012, a new highly pathogenic human coronavirus $(\mathrm{CoV})^{1}$, Middle East respiratory syndrome coronavirus (MERS-CoV), has emerged in Jeddah and Saudi Arabia and quickly spread to some European countries [1-3]. The virus causes symptoms similar to Severe Acute Respiratory Syndrome Coronavirus (SARS$\mathrm{CoV}$ ), yet involving an additional component of acute renal failure [4]. Until 15 May 2014, it has infected at least 572 people with a fatality rate of about $30 \%$ globally (World Health Organization, global alert and response, http://www.who.int/csr/don/2014_05_15_mers/en/). Recently, human-to-human transmission of MERS-CoV has been confirmed; albeit, a serological study of major

\footnotetext{
* Correspondence: fish3970@gmail.com; cychou@ym.edu.tw

${ }^{2}$ Department of Nephrology, Chang-Gung Memorial Hospital, Keelung 204, Taiwan

'Department of Life Sciences and Institute of Genome Sciences, National Yang-Ming University, Taipei 112, Taiwan
}

livestock suggested dromedary camels also to be a possible host $[5,6]$. Nevertheless, these findings indicate that the virus have the opportunity to spread globally and pose a significant threat to world health and the economy. Therefore, studies to understand the virus and to develop antiviral drugs or therapy are necessary and urgent.

Like other CoVs, the MERS-CoV nonstructural polyproteins (pp1a and pp1ab) are cleaved by two types of viral cysteine proteases, a main protease (EC 3.4.22.69) and a papain-like protease ( $\mathrm{PL}^{\mathrm{pro}}$ ) (EC 3.4.22.46) [7]. This processing is considered to be a suitable antiviral target because it is required for viral maturation. Unfortunately, initial screening of the existing SARS-CoV PL ${ }^{\text {pro }}$ inhibitor, a benzodioxolane derivative against MERS-CoV $\mathrm{PL}^{\mathrm{pro}}$, revealed no significant inhibition [7]. The difference represents the requirement of further understanding the MERS-

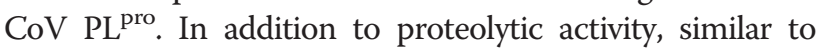
those of SARS-CoV, NL63-CoV and murine hepatitis virus, MERS-CoV $\mathrm{PL}^{\text {pro }}$ acts on both deubiquitination and ISG15-linked ISGylation [8-11]. As a viral deubiquitinating 
protease (DUB), MERS-CoV PL ${ }^{\text {pro }}$ is able to deubiquitinate interferon regulatory factor 3 (IRF3), which can prevent its nuclear translocation and suppress production of interferon $\beta$ [10]. These studies support the multifunctional nature of coronaviral $\mathrm{PL}^{\text {pro }}$. Recently, with the crystal structure of SARS-CoV PL ${ }^{\text {pro }}$ C112S mutant in complex with ubiquitin (Ub), we have demonstrated that $\mathrm{Ub}$ core (residue 1-72) makes mostly hydrophilic interactions with $\mathrm{PL}^{\text {pro }}$, while the Leu-Arg-Gly-Gly C-terminus of Ub is located in the catalytic cleft of $\mathrm{PL}^{\text {pro }}$, mimicking the P4-P1 residues [12]. This bound pattern is similar to that of the ubiquitin-specific proteases (USPs), one of the five distinct DUB families [13,14].

The MERS-CoV PL ${ }^{\text {pro }}$ domain in nsp3 of the pp1a proteins (residue 1484-1800) has been identified [7,10,15]. Like other $\mathrm{PL}^{\text {pro }}$, there is a catalytic triad consisting of the residues Cys1592, His1759 and Asp1774. Homology modeling suggests that MERS-CoV PL ${ }^{\text {pro }}$, similar to other known $\mathrm{PL}^{\text {pro }}$, may have a right-hand-like architecture constituted by palm, thumb, and fingers domains, although their sequence identity are only about 30\% [12]. Furthermore, MERS-CoV PL ${ }^{\text {pro }}$ is able to recognize and cleave at the LXGG consensus cleavage site, which is essential for most $\mathrm{CoV} \mathrm{PL}^{\mathrm{pro}}$-mediated processing [10]. Despite this large body of knowledge on MERS-CoV PL ${ }^{\text {pro }}$, in the absence of detailed structural and functional characterization, the molecular basis for its catalytic mechanism remains poorly unknown.

Here, we expressed and purified the MERS-CoV PL pro by $E$. coli with high yield and high purity. The secondary, tertiary and quaternary structure of MERS-CoV $\mathrm{PL}^{\text {pro }}$ was then investigated by circular dichroism (CD) spectroscopy, Tyr/Trp fluoresecence and analytical ultracentrifugation (AUC), respectively. The kinetic and DUB activity assays indicated that MERS-CoV PL ${ }^{\text {pro }}$ exhibits potent DUB activity but lower proteolytic activity, compared with SARS-CoV PL ${ }^{\text {pro }}$. The present study provides a foundation for understanding the structural and biochemical properties of coronaviral $\mathrm{PL}^{\text {pro }}$ family, which is applicable to develop strategies inhibiting $\mathrm{PL}^{\text {pro }}$ for the effective control of highly pathogenic coronaviral infection.

\section{Methods}

\section{Expression plasmid construction}

The sequence of MERS-CoV PL ${ }^{\text {pro }}$ (GenBank accession number NC_019843.2; polyprotein residues 1484-1800) was synthesized (MDBio Inc.), digested by NcoI-XhoI and then inserted into the pET-28a(+) vector (Novagen). In the construct, the $6 \mathrm{x}$ His tag was retained at the C-terminus. The reading frame was confirmed by sequencing.

\section{Expression and purification of MERS-CoV PL ${ }^{\text {pro }}$}

The expression vector was transformed into E. coli BL21 (DE3) cells (Novagen). For large scaled protein expression, cultures were grown in LB medium of 0.8 liter at $37^{\circ} \mathrm{C}$ for $4 \mathrm{~h}$, induced with $0.4 \mathrm{mM}$ isopropyl- $\beta{ }_{-}{ }$-thiogalactopyranoside, and incubated overnight at $20^{\circ} \mathrm{C}$. After centrifuging at $6,000 \mathrm{xg}$ at $4^{\circ} \mathrm{C}$ for $15 \mathrm{~min}$, the cell pellets were resuspended in lysis buffer $(20 \mathrm{mM}$ Tris, $\mathrm{pH} 8.5$, $250 \mathrm{mM} \mathrm{NaCl}, 5 \%$ glycerol, $0.2 \%$ Triton X-100, and $2 \mathrm{mM}$ $\beta$-mercaptoethanol) and then lysed by sonication. The crude extract was then centrifuged at $12,000 \mathrm{x}$ g at $4{ }^{\circ} \mathrm{C}$ for $25 \mathrm{~min}$ to remove the insoluble pellet. The supernatant was incubated with 1 -ml Ni-NTA beads at $4^{\circ} \mathrm{C}$ for $1 \mathrm{~h}$ and then loaded into an empty column. After allowing the supernatant to flow through, the beads were washed with washing buffer $(20 \mathrm{mM}$ Tris, $\mathrm{pH} 8.5,250 \mathrm{mM} \mathrm{NaCl}$, $8 \mathrm{mM}$ imidazole, and $2 \mathrm{mM} \beta$-mercaptoethanol), and the protein was eluted with elution buffer $(20 \mathrm{mM}$ Tris, $\mathrm{pH} 8.5,30 \mathrm{mM} \mathrm{NaCl}, 150 \mathrm{mM}$ imidazole, and $2 \mathrm{mM}$ $\beta$-mercaptoethanol). The protein was then loaded onto a S-100 gel-filtration column (GE Healthcare) equilibrated with running buffer (20 mM Tris, $\mathrm{pH} 8.5,100 \mathrm{mM} \mathrm{NaCl}$, and $2 \mathrm{mM}$ dithiothreitol). The purity of the fractions collected was analyzed by SDS-PAGE and the protein was concentrated to $30 \mathrm{mg} / \mathrm{ml}$ by Amicon Ultra-4 10-kDa centrifugal filter (Millipore).

\section{Circular dichroism spectroscopy}

CD spectra of the recombinant MERS-CoV PL ${ }^{\text {pro }}$ using a JASCO J-810 spectropolarimeter showed measurements from 250 to $190 \mathrm{~nm}$ at $20^{\circ} \mathrm{C}$ in $50 \mathrm{mM}$ phosphate $\mathrm{pH}$ 6.5. The protein concentration was $1.0 \mathrm{mg} / \mathrm{ml}$. In wavelength scanning, the width of the cuvette was $0.1 \mathrm{~mm}$. The far-UV CD spectrum data were analyzed with the CDSSTR program $[16,17]$. In this analysis, the $\alpha$-helix, $\beta$-sheet, and random coil were split. To estimate the goodness-of-fit, the normalized root mean square deviation was calculated.

\section{Spectrofluorimetric analysis}

The fluorescence spectra of the enzyme at $1 \mu \mathrm{M}$ were monitored in a Perkin-Elmer LS50B luminescence spectrometer at $25^{\circ} \mathrm{C}$. The excitation wavelength was set at $280 \mathrm{~nm}$, and the fluorescence emission spectrum was scanned from 300 to $400 \mathrm{~nm}$. Measurement in the maximal peak, intensity, and average emission wavelength were used to confirm the protein folding $[18,19]$.

\section{Analytical ultracentrifugation analysis}

The AUC experiments were performed on a XL-A analytical ultracentrifuge (Beckman Coulter) using an An-50 $\mathrm{Ti}$ rotor [12,19-22]. The sedimentation velocity experiments were performed using a double-sector epon charcoal-filled centerpiece at $20^{\circ} \mathrm{C}$ with a rotor speed of 42,000 rpm. Protein solutions of MERS-CoV PL ${ }^{\text {pro }}$ $(1.0 \mathrm{mg} / \mathrm{ml})(330 \mu \mathrm{l})$ and reference $(370 \mu \mathrm{l})$ solutions were loaded into the centerpiece, respectively. The absorbance 
at $280 \mathrm{~nm}$ was monitored in a continuous mode with a time interval of $300 \mathrm{~s}$ and a step size of $0.003 \mathrm{~cm}$. Multiple scans at different time intervals were then fitted to a continuous c(s) distribution model using the SEDFIT program [23]. All size-and-shape distributions were analyzed at a confidence level of $\mathrm{p}=0.95$ by maximal entropy regularization and a resolution $\mathrm{N}$ of 200 with sedimentation coefficients between 0 and $20 \mathrm{~S}$ or molar mass between 0 and $1000 \mathrm{kDa}$.

\section{Steady-state kinetic analysis}

The peptidyl substrate, Dabcyl-FRLKGGAPIKGV-Edans, was used to measure the enzymatic activity of MERS-CoV $\mathrm{PL}^{\mathrm{pro}}$ and its mutants throughout the course of the study as described [24]. Specifically, the enhanced fluorescence emission upon substrate cleavage was monitored at excitation and emission wavelengths of 329 and $520 \mathrm{~nm}$, respectively, in a PerkinElmer LS 50B luminescence spectrometer. Fluorescence intensity was converted to the amount of hydrolyzed substrate using a standard curve drawn from the fluorescence measurements of well-defined concentrations of Dabcyl-FRLKGG and APIKGV-Edans peptides in a 1:1 ratio. This will also correct for the inner filter effect of the substrate. For the kinetic analysis, the reaction mixture contained 4-50 $\mu \mathrm{M}$ peptide substrate in $50 \mathrm{mM}$ phosphate $\mathrm{pH} 6.5$ in a total volume of $1 \mathrm{~mL}$. After the addition of the enzyme to the reaction mixture, the increase in fluorescence was continuously monitored at $30^{\circ} \mathrm{C}$. The increase in fluorescence was linear for at least $3 \mathrm{~min}$, and thus the slope of the line represented the initial velocity $(v)$. The steady-state kinetic parameters of the enzyme were determined by fitting the Michaelis-Menten equation (eq. 1) to the initial velocity data

$$
v=\frac{k_{\text {cat }}[E][S]}{K_{m}+[S]}
$$

in which $k_{\text {cat }}$ is the rate constant, $[E]$ and $[S]$ denote the enzyme and substrate concentration, and $K_{\mathrm{m}}$ is the Michaelis-Menten constant for the interaction between the peptide substrate and the enzyme.

\section{Deubiquitination assay}

The fluorogenic substrate Ub-7-amino-4-trifluoro-methylcoumarin (Ub-AFC) (Boston Biochem) added at 0.5 or $1.0 \mu \mathrm{M}$ to $50 \mathrm{mM}$ phosphate $\mathrm{pH} 6.5$ was used for deubiquitination assays as described [12]. The enzymatic activity at $30^{\circ} \mathrm{C}$ was determined by continuously monitoring the fluorescence emission and excitation wavelength of 350 and $485 \mathrm{~nm}$, respectively.

\section{Results and discussion \\ Recombinant MERS-CoV PL ${ }^{\text {pro }}$ preparation}

To date, there are still no studies describing the expression and purification of MERS-CoV PL pro proteins. In the present study, the expression vector was constructed and then various E. coli. strains such as BL21 (DE3) STAR (Invitrogen) and Rosetta (DE3) (Novagen) were used to explore heterologous expression of MERS-CoV $\mathrm{PL}^{\mathrm{pro}}$. Finally, it was found that the STAR strain showed

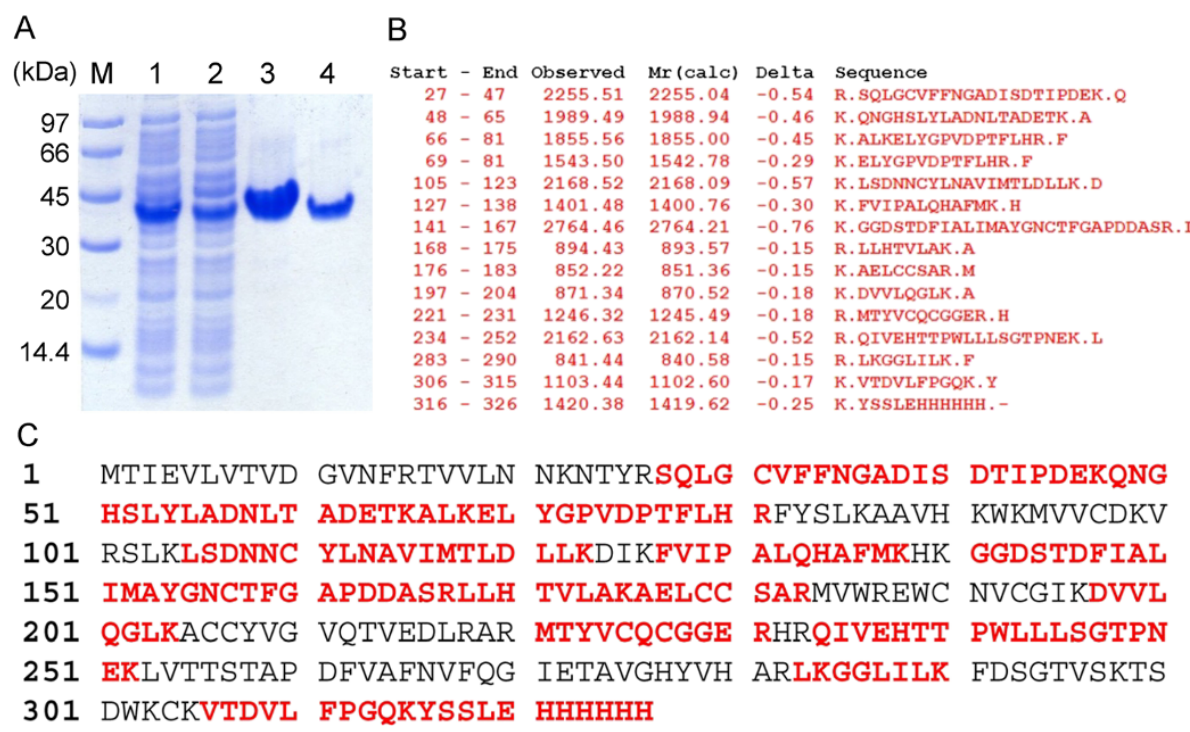

Figure 1 Expression and purification of recombinant MERS-CoV PL ${ }^{\text {pro }}$. (A) Protein identification by SDS-PAGE. M: molecular marker. Lane 1-4: cytoplasmic fraction, flow-through, elution from the nickel affinity column and protein fraction from S-100 gel-filtration column. (B) and (C) Protein sequence identification by mass spectrometry. The $\mathrm{PL}^{\text {pro }}$ was digested by trypsin and then analyzed by MALDI mass spectrometry. There are 15 matched peptides observed (B) and 60\% sequence coverage are shown in bold red (C). 
Table 1 Purification of MERS-CoV PL ${ }^{\text {pro }}$ from E. coli

\begin{tabular}{llllll}
\hline Step & Total protein $(\mathbf{m g})$ & Total activity $\left(\mathbf{U}^{\mathbf{a}}\right)$ & Specific activity $(\mathbf{U} / \mathbf{m g}$ protein) & Purification (-fold) & Recovery (\%) \\
\hline Cytoplasmic fraction & 426 & 340 & 0.80 & 1 & 100 \\
Ni affinity chromatography & 64.4 & 221 & 3.43 & 4.3 & 65 \\
Gel-filtration by S-100 column & 41.9 & 168 & 4.01 & 5.0 & 49.4
\end{tabular}

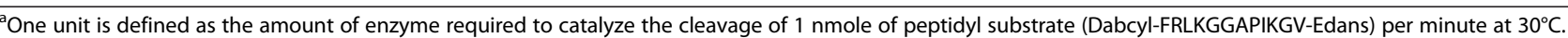

the best expression efficiency. After expressing the protein in $E$. coli and purification by nickel affinity chromatography and gel-filtration, the purity of recombinant $\mathrm{PL}^{\mathrm{pro}}$ was about $99 \%$ (Figure 1A). The size of the recombinant MERS-CoV PL ${ }^{\text {pro }}$ was found to be between 30 and $45 \mathrm{kDa}$, which conforms to the theoretical mass $(36.5 \mathrm{kDa})$. The typical yield was about $42 \mathrm{mg}$ after purification from 0.8 liter of $E$. coli culture (Table 1). After gel-filtration chromatography, the specific proteolytic activity of $\mathrm{PL}^{\text {pro }}$ was $4 \mathrm{U} / \mathrm{mg}$, increased by 5 -fold, with $49.4 \%$ recovery rate.

Furthermore, the recombinant MERS-CoV PL pro was digested by trypsin and then analyzed by MALDI mass spectrometry to confirm the amino acid sequence (Additional file 1: Figure S1). The molecular weight of fifteen peptides, which covered $60 \%$ amino acid sequence, was observed and confirmed (Figure 1B and Figure 1C). It indicated that our expression and purification of MERS$\mathrm{CoV} \mathrm{PL}^{\text {pro }}$ by $E$. coli is successful. For convenience, in the present studies, the MERS-CoV PL ${ }^{\text {pro }}$ domain (polyprotein 1a 1484-1800) is numbered to residue 2 to 317 , while the first residue is a methionine.

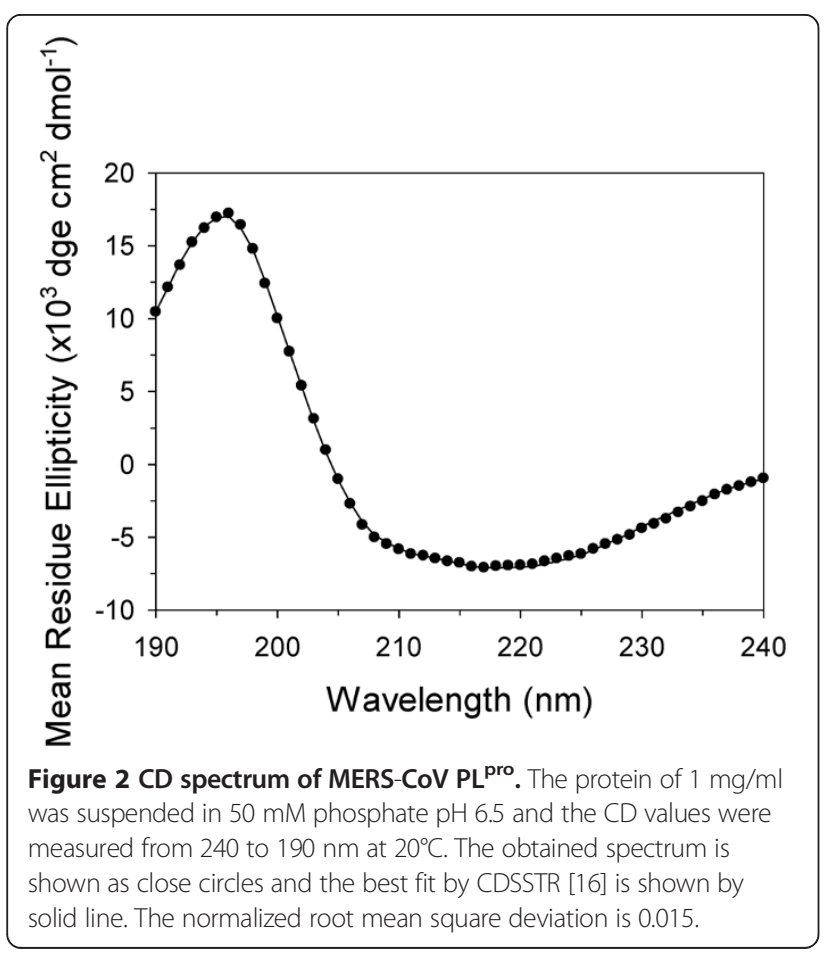

\section{Secondary, tertiary and quaternary structure analysis of MERS-CoV PL ${ }^{\text {pro }}$}

Next, secondary, tertiary and quaternary structures of MERS-CoV PL $^{\text {pro }}$ were investigated, respectively. CD measurement displayed a spectrum which shows negative ellipticity between 240 and $205 \mathrm{~nm}$ and positive between 205 and $190 \mathrm{~nm}$ (Figure 2). After analyzed by CDSSTR method [16], the best-fit result showed that MERS-CoV PL ${ }^{\text {pro }}$ has $23 \%$ of $\alpha$-helix, $31 \%$ of $\beta$-sheet, and $46 \%$ of random coil. The consist is close to that of SARS-CoV PL ${ }^{\text {pro }}$ (pdb code: $4 \mathrm{MOW}$ ) by X-ray crystallography, which has $26 \%$ of $\alpha$-helix, $36 \%$ of $\beta$-sheet, and $38 \%$ of random coil [12]. It suggests that both $\mathrm{PL}^{\text {pro }}$ may have a similar scaffold.

The Tyr/Trp fluorescence of MERS-CoV $\mathrm{PL}^{\text {pro }}$ at the phosphate buffer without or with $9 \mathrm{M}$ urea were also identified (Figure 3). The measurement indicated that the fluorescent intensity of native $\mathrm{PL}^{\text {pro }}$ (Figure 3, close circles) shows a 70\% increase, as compared with that of the denatured form in urea (Figure 3, open circles). On the other hand, the fluorescence emission spectrum of the native MERS-CoV PL ${ }^{\text {pro }}$ shows a maximum at $336 \mathrm{~nm}$, while that of the unfolded one shifts to $340 \mathrm{~nm}$. The tendency is similar to that of SARS-CoV $\mathrm{PL}^{\text {pro }}[19]$ and suggests a folded structure. Next, we also performed AUC experiments to characterize the

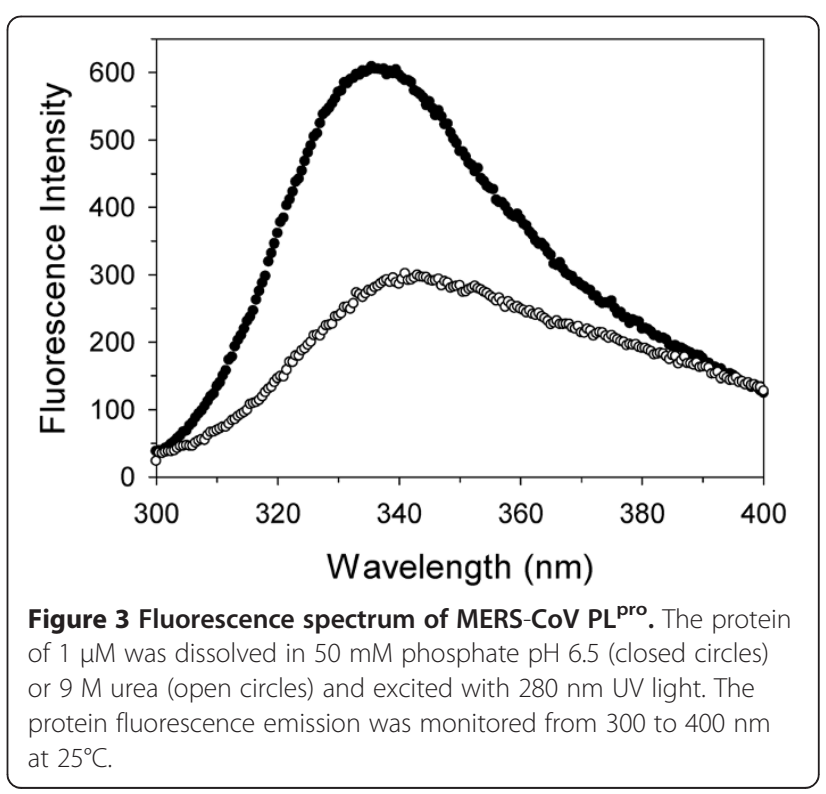


quaternary structure of MERS-CoV PL ${ }^{\text {pro }}$. Figure $4 \mathrm{~A}$ shows a typical absorbance trace at $280 \mathrm{~nm}$ of the $\mathrm{PL}^{\text {pro }}$ during the experiment. After fitting the signals to a continuous size-distribution model, it was clear that the $\mathrm{PL}^{\text {pro }}$ was monomeric with a sedimentation coefficient of $2.8 \mathrm{~S}$ and molar mass of $35.5 \mathrm{kDa}$ (Figure $4 \mathrm{~B}$ and Figure $4 \mathrm{C}$ ), consistent with that for SARS-CoV PL pro $[12,19]$. All of these biophysical observation confirmed that the PL ${ }^{\text {pro }}$ of MERS-CoV and SARS-CoV should have a very similar structure; albeit they only show $30 \%$ sequence identity and 50\% similarity [12]. Recent studies hypothesized that the homology model of MERS-CoV $\mathrm{PL}^{\text {pro }}$, like other coronaviral $\mathrm{PL}^{\text {pro }}$, is a right-handlike architecture consisting of palm, thumb and fingers domains [10,25].

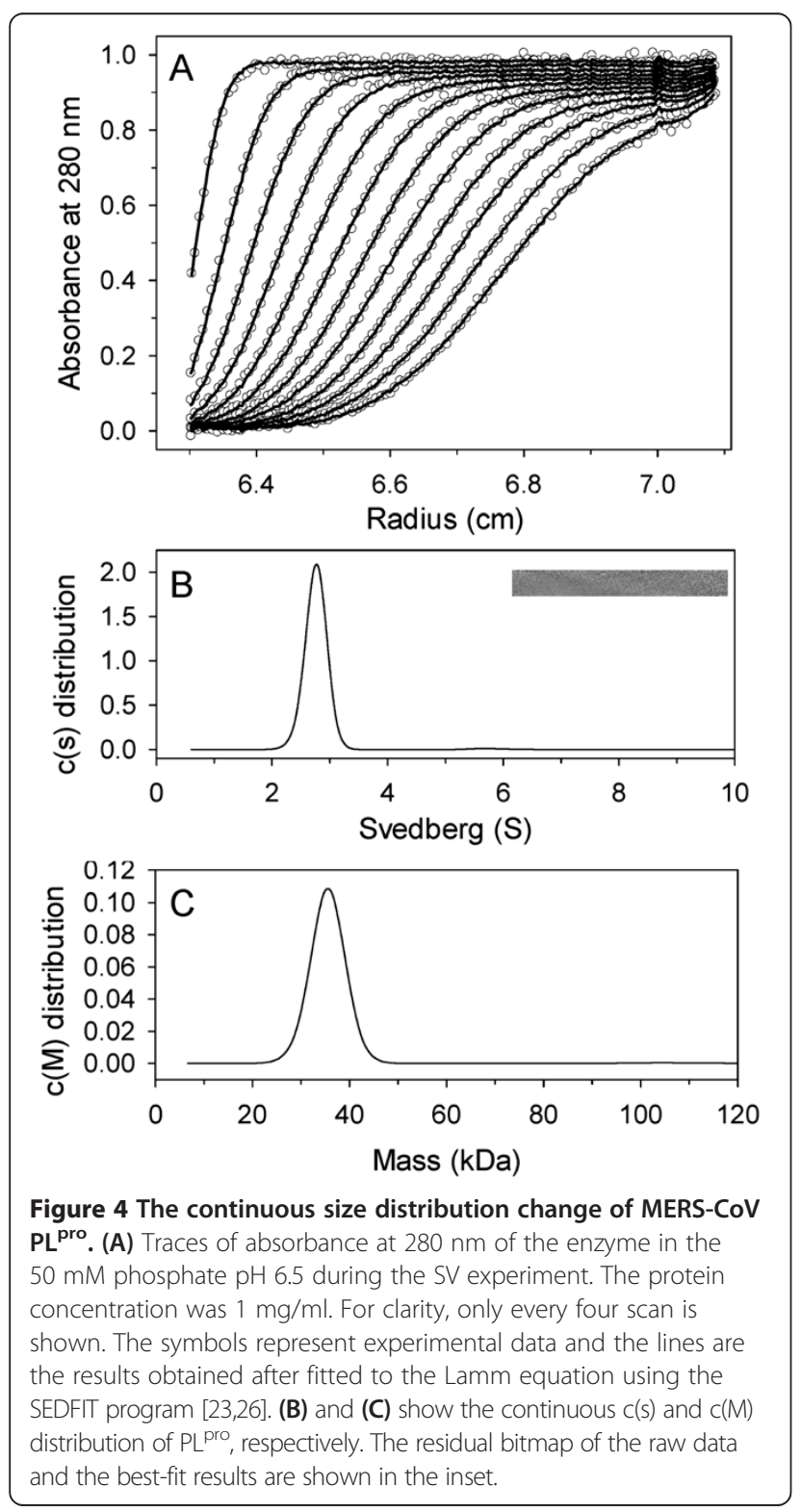

Proteolytic activity of MERS-CoV PL ${ }^{\text {pro }}$

Besides the structural similarity, previous studies have suggested that MERS-CoV PL ${ }^{\text {pro }}$ is also a multifunctional enzyme with protease, deubiquitinating and interferon antagonist activities [10]. MERS-CoV PL ${ }^{\text {pro }}$ has a catalytic triad which is able to recognize and cleave at LXGG consensus cleavage sites; however, the detail enzyme kinetic mechanism is not known. Here we used the peptidyl substrate, Dabcyl-FRLKGGAPIKGV-Edans, to measure the proteolytic activity of MERS-CoV PL ${ }^{\text {pro }}$ (Figure 5A and Table 2). Interestingly, compared with that
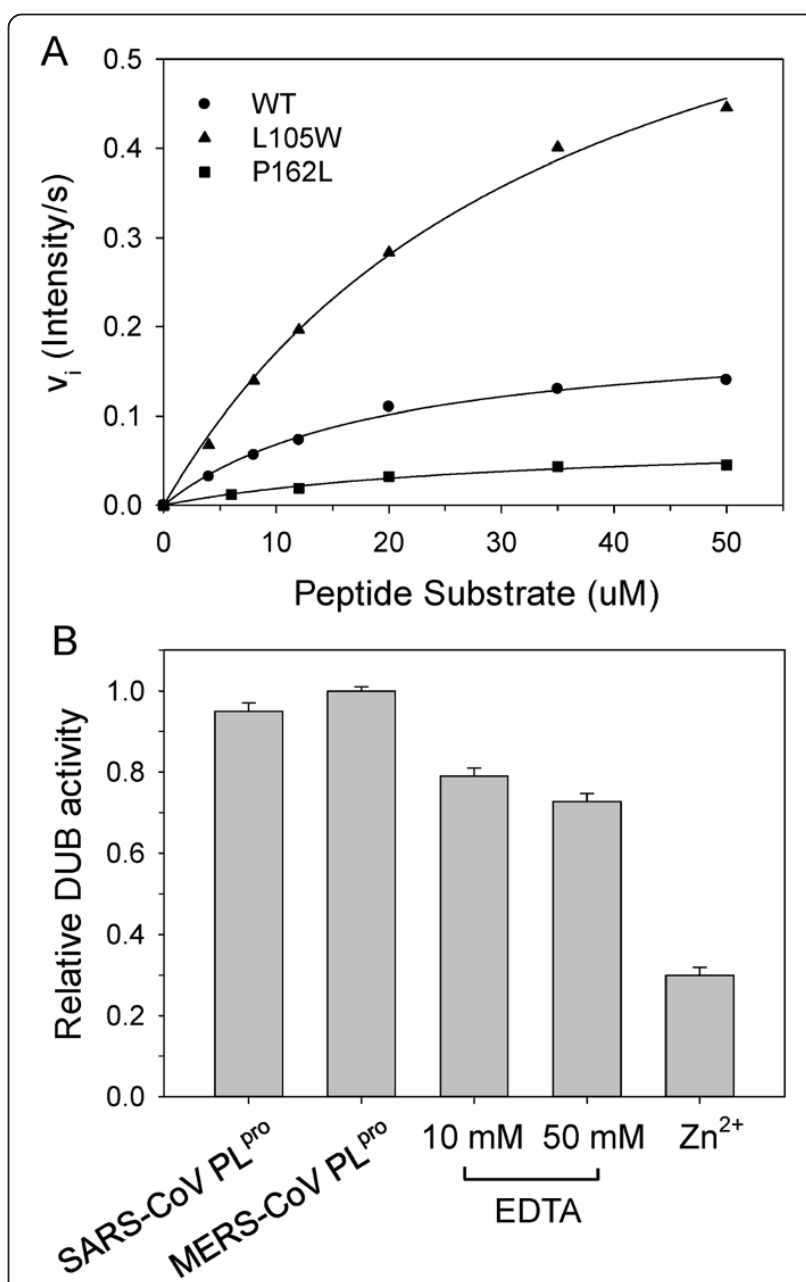

Figure 5 Proteolytic and DUB activity assay of MERS-CoV PL pro and its mutants. Panel (A) shows the plot of initial velocities versus the concentration of peptidyl substrate, Dabcyl-FRLKGGAPIKGV-Edans. The concentration of the wild-type MERS-CoV PL ${ }^{\text {pro }}$ (by circles), the L105W (by triangles) and P162L mutants (by squares) was 1, 0.1 and

$10 \mu \mathrm{M}$, respectively. The line represented the best-fit results according to the Michaelis-Menten equation (Eq. 1). The kinetic parameters derived are shown in Table 2. (B) DUB activity analysis. The fluorogenic substrate Ub-AFC (1 $\mu \mathrm{M})$ was used as the substrate. For comparison, both DUB activity of SARS-CoV and MERS-CoV PL pro was tested. The protein concentration was $0.17 \mu \mathrm{M}$. Besides, the inhibition of MERS-CoV

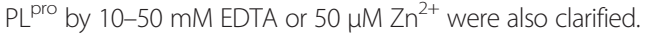


Table 2 The kinetic parameters and DUB activity of MERS-CoV PL ${ }^{\text {pro }}$

\begin{tabular}{|c|c|c|c|c|}
\hline \multirow[t]{2}{*}{ Proteins } & \multicolumn{3}{|c|}{ Peptide cleavage } & \multirow{2}{*}{$\begin{array}{l}\text { Deubiquitination } \\
\text { Activity } \\
\text { (Intensity/s) }^{\mathbf{b}}\end{array}$} \\
\hline & $\begin{array}{l}\mathrm{K}_{\mathrm{m}} \\
(\mu \mathrm{M})^{\mathrm{a}}\end{array}$ & $\begin{array}{l}k_{\text {cat }} \\
\left(10^{-2} s^{-1}\right)^{a}\end{array}$ & $\begin{array}{l}k_{\text {cat }} / K_{m} \\
\left(10^{-3} s^{-1} \mu M^{-1}\right)\end{array}$ & \\
\hline \multicolumn{5}{|l|}{$\begin{array}{l}\text { MERS-CoV } \\
\text { PL }^{\text {pro }}\end{array}$} \\
\hline Wild-type & $19.2 \pm 2.6$ & $0.4 \pm 0.02$ & $0.2 \pm 0.03$ & $0.11 \pm 0.02$ \\
\hline $\begin{array}{l}\text { L105W } \\
\text { mutant }\end{array}$ & $35.7 \pm 3.8$ & $16.5 \pm 0.9$ & $4.6 \pm 0.6$ & $0.11 \pm 0.01$ \\
\hline $\begin{array}{l}\text { P162L } \\
\text { mutant }\end{array}$ & $30.8 \pm 8.0$ & $0.01 \pm 0.001$ & $0.003 \pm 0.001$ & $0.004 \pm 0.001$ \\
\hline $\begin{array}{l}\text { SARS-CoV } \\
\text { PL }^{\text {pro }}\end{array}$ & $25.2 \pm 5.1^{\mathrm{c}}$ & $11 \pm 2^{c}$ & $4.4 \pm 1.2^{c}$ & $0.12 \pm 0.02$ \\
\hline
\end{tabular}

${ }^{\text {a Kinetic data of MERS-CoV PL }}{ }^{\text {pro }}$ and its mutants were fitted to the MichaelisMenten equation (Eq. 1). The $R_{\text {sqr }}$ were from 0.986 to 0.997 , respectively. All the assays were repeated several times to ensure reproducibility.

bixed concentrations of Ub-AFC $(0.5 \mu \mathrm{M})$ and PL ${ }^{\text {pro }}(0.17 \mu \mathrm{M})$ were used.

'The values were from our previous studies [24].

of SARS-CoV, MERS-CoV PL ${ }^{\text {pro }}$ is less active, with a 22fold loss in $\mathrm{k}_{\text {cat }} / \mathrm{K}_{\mathrm{m}}$, as a result of a 27.5 -fold loss in $\mathrm{k}_{\text {cat }}$ and 1.3-fold loss in $\mathrm{K}_{\mathrm{m}}$. According to the sequence alignment and homology modeling, most important residues for the catalysis, including the catalytic triad, Cys110His277-Asp292 and the residues for substrate P4-P1 binding, Asp164, Pro249, and Gly276 (Asp165, Pro249, and Gly272 in SARS-CoV PL ${ }^{\text {pro }}$ ) are highly conserved (Figure 6) $[10,12]$. Previous studies have confirmed that Y265F mutant of SARS-CoV PL ${ }^{\text {pro }}$ still maintained comparable proteolytic activity with the wild-type [12]. It indicates that the equivalent residue in MERS-CoV PL ${ }^{\text {pro }}$, Phe268, is able to make a hydrophobic contact with the substrate P4 residue (Ub-Leu73). Furthermore, although different to the residue Tyr269 of SARS-CoV PL ${ }^{\text {pro }}$, the equivalent residue Glu272 of MERS-CoV PL ${ }^{\text {pro }}$, whose carboxyl group can point toward outside the hydrophobic pocket, may not interfere the binding of substrate P4 residue (Figure 6). By contrast, as a putative oxyanion bound residue (Tyr107 in SARSCoV PL ${ }^{\text {pro }}$ ) [15], the equivalent residue Leu105 of MERS$\mathrm{CoV} \mathrm{PL}^{\text {pro }}$ cannot provide any hydrogen bonding interaction with oxyanion (Figure 6). It will disfavor the formation of tetrahedral intermediate. Otherwise, different to the Leu163 of SARS-CoV PL ${ }^{\text {pro }}$, the distinct circular structure of the equivalent residue Pro162 of MERS-CoV PL ${ }^{\text {pro }}$ may be too short to hover above the active site for substrate binding and serve to enhance the nucleophilicity of the catalytic triad residue, Cys110 (Figure 6). These two point mutations in MERS-CoV PL ${ }^{\text {pro }}$ may significantly lower the catalytic efficiency.

To verify this, we produced the L105W and P162L mutants of MERS-CoV PL ${ }^{\text {pro }}$, and our kinetic data showed that the L105W mutant has a 23-fold increase in activity measured based on $\mathrm{k}_{\text {cat }} / \mathrm{K}_{\mathrm{m}}$, as a result of a 41-fold increase in $k_{\text {cat }}$ and 1.9-fold increase in $K_{m}$ (Figure 5A and Table 2). The results conform to our prediction. However, in contrast, the P162L mutant has a 67-fold loss in $\mathrm{k}_{\text {cat }} / \mathrm{K}_{\mathrm{m}}$, as a result of a 40 -fold loss in $\mathrm{k}_{\text {cat }}$ and 1.6-fold increase in $\mathrm{K}_{\mathrm{m}}$ (Figure 5A and Table 2). It suggests the requirement of the Proline residue in this site, although the reason is still not known. Nevertheless, the significant activity recovery by $\mathrm{L} 105 \mathrm{~W}$ mutation confirms the essential role of this residue on coronaviral $\mathrm{PL}^{\text {pro }}$ catalysis. Theoretically, $\mathrm{PL}^{\text {pro }}$ with lower proteolytic

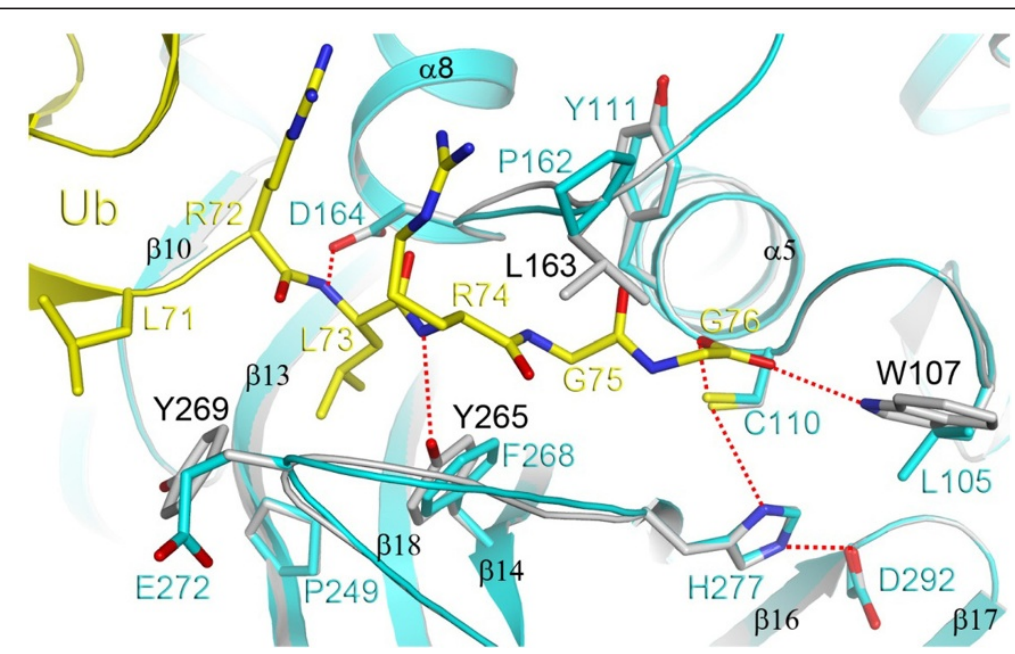

Figure 6 Putative active site of MERS-CoV PL ${ }^{\text {pro }}$. The model structure of MERS-CoV PL ${ }^{\text {pro }}$ (in cyan) was generated by SWISS-MODEL [27] and then overlaid with the structure of SARS-CoV PL pro (in grey) in complex with Ub (in yellow) (PDB code: 4MOW). The residues are shown as sticks

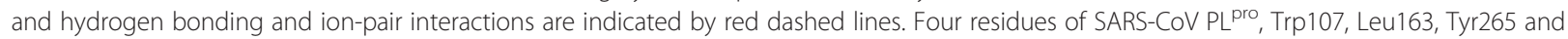
Tyr269, are labeled in black. The figure was produced using PyMol (http://www.pymol.org). 
activity may result in late maturation of viral nsp1, nsp2, and nsp3 proteins; nonetheless, its influence on MERS$\mathrm{CoV}$ remains unknown.

\section{DUB activity of MERS-CoV PL ${ }^{\text {pro }}$}

To characterize the DUB activity of MERS-CoV PL pro, the fluorogenic substrate Ub-AFC was used. Interestingly, in contrast with its rather low proteolytic activity, MERS-CoV PL ${ }^{\text {pro }}$ shows comparable DUB activity to SARS-CoV PL ${ }^{\text {pro }}$ (Table 2 and Figure 5B). It suggests that the two $\mathrm{PL}^{\text {pro }}$ may show similar binding ability to the Ub core domain (residue 1-72). However, it is inconsistent with our previous observation on the structure of SARS-CoV PL ${ }^{\text {pro }}$ in complex with Ub [12]. As mimicking the equivalent residue of MERS-CoV $\mathrm{PL}^{\text {pro }}$, the arginine mutation of a key residue for Ub core domain binding, Glu168, can result in unstable binding of SARS-CoV PL ${ }^{\text {pro }}$ and Ub and significant loss of DUB activity [12]. To verify this inconsistency, a structure of MERS$\mathrm{CoV} \mathrm{PL}^{\text {pro }}$ in complex with $\mathrm{Ub}$ is quite necessary.

Structural characterization of type 1 and type $2 \mathrm{PL}^{\mathrm{pro}}$ have revealed that there are four cysteine residues coordinating to a zinc ion within the fingertips region in the finger domain $[25,28]$. Remove of zinc from SARS$\mathrm{CoV} \mathrm{PL}^{\text {pro }}$ will cause the tertiary structure more unstable and lead to less active [19]. Based on sequence alignment, MERS-CoV PL ${ }^{\text {pro }}$ also has four cysteine residues (Cys190, Cys193, Cys225 and Cys227) on the corresponding position. Here the DUB activity of MERS-CoV $\mathrm{PL}^{\text {pro }}$ in various EDTA was examined to delineate the possible metal ion effect. The activity was $79 \%$ in $10 \mathrm{mM}$, and $72 \%$ left in $50 \mathrm{mM}$ EDTA (Figure 5B). These results suggest the existence of endogenous metal ion, which is beneficial for its DUB activity. By the way, it has been clarified that exogenous zinc ion can efficiently inhibit SARS$\mathrm{CoV}$ PL ${ }^{\text {pro }}$ with the $\mathrm{IC}_{50}$ value of $1.3 \mu \mathrm{M}[24,29]$. Here we also confirmed the potent inhibitory effect of zinc ion on MERS-CoV PL ${ }^{\text {pro }}$ (Figure 5B); whereas the mechanism of this inhibition by zinc is not yet understood.

\section{Conclusions}

In summary, following our protocol, active MERS-CoV $\mathrm{PL}^{\text {pro }}$ can be expressed by E. coli and purified with high yield and high purity. The secondary, tertiary and quaternary structural studies concluded that MERS-CoV $\mathrm{PL}^{\text {pro }}$ has a similar scaffold to other coronaviral $\mathrm{PL}^{\text {pro }}$, as a right-hand-like architecture consisting of palm, thumb and fingers domains. The result of functional assay indicated that MERS-CoV PL ${ }^{\text {pro }}$ exhibits potent DUB activity but rather low proteolytic activity. A natural mutation, Leu105, is the major reason for this difference. The present study not only demonstrates the structural and functional characterization of MERS-CoV PL ${ }^{\text {pro }}$, but provides a foundation for further understanding the coronaviral $\mathrm{PL}^{\text {pro }}$ family, which is an ideal antiviral target. Next, with pure protein and effective proteolytic activity assay, potent inhibitors of MERS-CoV PL ${ }^{\text {pro }}$ can be high throughput screened and identified.

\section{Additional file}

Additional file 1: Figure S1. Mass spectrometry of trypsin-digested peptides of the recombinant MERS-CoV PLpro protein. The red peaks show the signals of the peptides with correct mass, while the blue ones show the signals of the peptides with oxidation. X-axis indicates the $\mathrm{m} / \mathrm{z}$ ratio and $Y$-axis shows the absorbance intensity.

\section{Abbreviations}

${ }^{1}$ AFC: 7-amino-4-trifluoro-methylcoumarin; AUC: analytical ultracentrifugation; $\beta$-ME: $\beta$-mercaptoethanol; CD: circular dichroism; CoV: coronavirus; DUB: deubiquitinating protease; IRF3: interferon regulatory factor 3; MERSCoV: Middle East respiratory syndrome coronavirus; PCR: polymerase chain reaction; $\mathrm{PL}^{\text {pro: }}$ : papain-like protease; SARS-CoV: severe acute respiratory syndrome coronavirus; SV: sedimentation velocity; Ub: ubiquitin.

\section{Competing interests}

The authors declare that they have no competing interests.

\section{Authors' contributions}

MHL carried out most experiments and analyzed the kinetic data. SJC expressed and purified the protein. CCC and $\mathrm{CHL}$ acquired and analyzed the data by mass spectrometry. SCC amplified the cDNA and constructed the expression plasmid. KWC participated in experimental design on structural analysis. CYS and CYC conceived the whole study, participated in experimental design and wrote the manuscript. All authors read and approved the final manuscript.

\section{Acknowledgements}

This research was supported by grants from National Science Council, Taiwan (98-2320-B-010-026-MY3 and 101-2320-B-010-061) to CYC and CGMH-NYMU Joint Research Grant (CMRPG2D0211) to CYC and CYS. We also thank NYMU for its financial support (Aim for Top University Plan from Ministry of Education).

Received: 23 April 2014 Accepted: 19 May 2014

Published: 4 June 2014

\section{References}

1. Anderson LJ, Baric RS: Emerging human coronaviruses-disease potential and preparedness. N Engl J Med 2012, 367(19):1850-1852.

2. Chan JF, Li KS, To KK, Cheng VC, Chen H, Yuen KY: Is the discovery of the novel human betacoronavirus 2c EMC/2012 (HCoV-EMC) the beginning of another SARS-like pandemic? J Infect 2012, 65(6):477-489.

3. Zaki AM, van Boheemen S, Bestebroer TM, Osterhaus AD, Fouchier RA: Isolation of a novel coronavirus from a man with pneumonia in Saudi Arabia. N Engl J Med 2012, 367(19):1814-1820.

4. Eckerle I, Muller MA, Kallies S, Gotthardt DN, Drosten C: In-vitro renal epithelial cell infection reveals a viral kidney tropism as a potential mechanism for acute renal failure during Middle East Respiratory Syndrome (MERS) Coronavirus infection. Virol J 2013, 10:359.

5. Assiri A, McGeer A, Perl TM, Price CS, Al Rabeeah AA, Cummings DA, Alabdullatif ZN, Assad M, Almulhim A, Makhdoom H, Madani H, Alhakeem R, Al-Tawfiq JA, Cotten M, Watson SJ, Kellam P, Zumla Al, Memish ZA, Team KM-Cl: Hospital outbreak of Middle East respiratory syndrome coronavirus. N Engl J Med 2013, 369(5):407-416.

6. Reusken CB, Haagmans BL, Muller MA, Gutierrez C, Godeke GJ, Meyer B, Muth D, Raj VS, Smits-De VL, Corman VM, Drexler JF, Smits SL, El Tahir YE, De Sousa R, van Beek J, Nowotny N, van Maanen K, Hidalgo-Hermoso E, Bosch BJ, Rottier P, Osterhaus A, Gortazar-Schmidt C, Drosten C, Koopmans MP: Middle East respiratory syndrome coronavirus neutralising serum antibodies in dromedary camels: a comparative serological study. Lancet Infect Dis 2013, 13(10):859-866. 
7. Kilianski A, Mielech AM, Deng X, Baker SC: Assessing activity and inhibition of Middle East respiratory syndrome coronavirus papain-like and 3C-like proteases using luciferase-based biosensors. J Virol 2013, 87(21):11955-11962.

8. Chen Z, Wang Y, Ratia K, Mesecar AD, Wilkinson KD, Baker SC: Proteolytic processing and deubiquitinating activity of papain-like proteases of human coronavirus NL63. J Virol 2007, 81(11):6007-6018.

9. Clementz MA, Chen Z, Banach BS, Wang Y, Sun L, Ratia K, Baez-Santos YM, Wang J, Takayama J, Ghosh AK, Li K, Mesecar AD, Baker SC: Deubiquitinating and interferon antagonism activities of coronavirus papain-like proteases. $J$ Virol 2010, 84(9):4619-4629.

10. Yang $X$, Chen $X$, Bian G, Tu J, Xing $Y$, Wang $Y$, Chen Z: Proteolytic processing, deubiquitinase and interferon antagonist activities of Middle East respiratory syndrome coronavirus papain-like protease. J Gen Virol 2014, 95(Pt 3):614-626.

11. Zheng D, Chen G, Guo B, Cheng G, Tang H: PLP2, a potent deubiquitinase from murine hepatitis virus, strongly inhibits cellular type I interferon production. Cell Res 2008, 18(11):1105-1113.

12. Chou CY, Lai HY, Chen HY, Cheng SC, Cheng KW, Chou YW: Structural basis for catalysis and ubiquitin recognition by the Severe acute respiratory syndrome coronavirus papain-like protease. Acta Crystallogr D Biol Crystallogr 2014, 70(Pt 2):572-581.

13. Hu M, Li P, Song L, Jeffrey PD, Chenova TA, Wilkinson KD, Cohen RE, Shi Y: Structure and mechanisms of the proteasome-associated deubiquitinating enzyme USP14. EMBO J 2005, 24(21):3747-3756.

14. Renatus M, Parrado SG, D'Arcy A, Eidhoff U, Gerhartz B, Hassiepen U, Pierrat B, Riedl R, Vinzenz D, Worpenberg S, Kroemer M: Structural basis of ubiquitin recognition by the deubiquitinating protease USP2. Structure 2006, 14(8):1293-1302.

15. Mielech AM, Kilianski A, Baez-Santos YM, Mesecar AD, Baker SC: MERS-CoV papain-like protease has delSGylating and deubiquitinating activities. Virology 2014, 450-451:64-70.

16. Sreerama N, Woody RW: Estimation of protein secondary structure from circular dichroism spectra: comparison of CONTIN, SELCON, and CDSSTR methods with an expanded reference set. Anal Biochem 2000, 287(2):252-260

17. Whitmore $L$, Wallace BA: DICHROWEB, an online server for protein secondary structure analyses from circular dichroism spectroscopic data. Nucleic Acids Res 2004, 32(Web Server issue):W668-673.

18. Chang HP, Chou CY, Chang GG: Reversible unfolding of the severe acute respiratory syndrome coronavirus main protease in guanidinium chloride. Biophys J 2007, 92(4):1374-1383.

19. Chou YW, Cheng SC, Lai HY, Chou CY: Differential domain structure stability of the severe acute respiratory syndrome coronavirus papainlike protease. Arch Biochem Biophys 2012, 520(2):74-80.

20. Cheng SC, Chang GG, Chou CY: Mutation of Glu-166 blocks the substrateinduced dimerization of SARS coronavirus main protease. Biophys J 2010, 98(7):1327-1336

21. Hsieh $\mathrm{YH}$, Chou $\mathrm{CY}$ : Structural and functional characterization of human apolipoprotein E 72-166 peptides in both aqueous and lipid environments. J Biomed Sci 2011, 18:4.

22. Wu CG, Cheng SC, Chen SC, Li JY, Fang YH, Chen YH, Chou CY: Mechanism for controlling the monomer-dimer conversion of SARS coronavirus main protease. Acta Crystallogr D Biol Crystallogr 2013, 69(Pt 5):747-755.

23. Schuck P: Size-distribution analysis of macromolecules by sedimentation velocity ultracentrifugation and Lamm equation modeling. Biophys J 2000, 78:1606-1619.

24. Chou CY, Chien CH, Han YS, Prebanda MT, Hsieh HP, Turk B, Chang GG, Chen $X$ : Thiopurine analogues inhibit papain-like protease of severe acute respiratory syndrome coronavirus. Biochem Pharmacol 2008, 75(8):1601-1609.

25. Wojdyla JA, Manolaridis I, van Kasteren PB, Kikkert M, Snijder EJ Gorbalenya $A E$, Tucker PA: Papain-like protease 1 from transmissible gastroenteritis virus: crystal structure and enzymatic activity toward viral and cellular substrates. J Virol 2010, 84(19):10063-10073.

26. Brown PH, Schuck P: Macromolecular size-and-shape distributions by sedimentation velocity analytical ultracentrifugation. Biophys $J 2006$, 90(12):4651-4661.

27. Benkert $\mathrm{P}$, Biasini M, Schwede T: Toward the estimation of the absolute quality of individual protein structure models. Bioinformatics 2011, 27(3):343-350
28. Ratia K, Saikatendu KS, Santarsiero BD, Barretto N, Baker SC, Stevens RC, Mesecar AD: Severe acute respiratory syndrome coronavirus papain-like protease: structure of a viral deubiquitinating enzyme. Proc Natl Acad Sci US A 2006, 103(15):5717-5722.

29. Han YS, Chang GG, Juo CG, Lee HJ, Yeh SH, Hsu JT, Chen X: Papain-like protease 2 (PLP2) from severe acute respiratory syndrome coronavirus (SARS-CoV): expression, purification, characterization, and inhibition. Biochemistry 2005, 44(30):10349-10359.

doi:10.1186/1423-0127-21-54

Cite this article as: Lin et al:: Structural and functional characterization of MERS coronavirus papain-like protease. Journal of Biomedical Science 2014 21:54.

\section{Submit your next manuscript to BioMed Central and take full advantage of:}

- Convenient online submission

- Thorough peer review

- No space constraints or color figure charges

- Immediate publication on acceptance

- Inclusion in PubMed, CAS, Scopus and Google Scholar

- Research which is freely available for redistribution 